\title{
Las capacidades dinámicas: análisis de las empresas exportadoras en América Latina
}

\section{Dynamic capabilities: analysis of export companies in Latin America}

\author{
Diana Carolina Feijoo-Pardo \\ Mayiya Lisbeth González-Illescas \\ Universidad Técnica de Machala, Ecuador
}

Autor para correspondencia: dcfeijoo_est@utmachala.edu.ec; mlgonzalez@utmachala.edu.ec Fecha de recepción: 27 de agosto del 2019 - Fecha de aceptación: 13 de diciembre del 2019

\section{Resumen}

Las capacidades dinámicas permiten a las empresas estar preparadas ante el cambio constante de su entorno. En el caso de las empresas exportadoras, el monitoreo de las transformaciones de los mercados es una actividad clave para levantar alertas sobre los cambios que debe realizar para brindar respuestas acordes a las nuevas exigencias de la demanda. Este trabajo tiene como propósito analizar las capacidades dinámicas que han desarrollado varias empresas exportadoras de América Latina, a partir de la revisión de los trabajos de otros autores que han profundizado en la realidad de cada organización. Se aplica un enfoque cualitativo y los métodos teóricos histórico lógico, analítico sintético y el inductivo deductivo. Los principales hallazgos de las capacidades dinámicas reflejan la evolución del análisis estratégico de la empresa y de los factores que contribuyen a fortalecer las ventajas competitivas sostenibles y la capacidad de absorber las buenas prácticas externas, la orientación a innovaciones que se derivan en mejoras de procesos, reducción de costos, ampliación de una visión empresarial y de conocimientos que fortalecen el capital intelectual para la toma de decisiones.

Palabras claves: capacidades dinámicas; mercados dinámicos; aprendizaje organizacional; ventajas competitivas; empresas exportadoras

\begin{abstract}
Dynamic capabilities allow companies to be prepared for the constant change of their environment. In the case of exporting companies, the monitoring of market transformations is a key activity to raise alerts about the changes that must be made to provide answers according to the new demands of the demand. The purpose of this work is to analyze the dynamic capacities that several exporting companies in Latin America have developed, based on the review of the work of other authors that have deepened the reality of each organization. A qualitative approach and theoretical historical, synthetic analytical and deductive inductive theoretical methods are applied. The main findings of dynamic capabilities reflect the evolution of the strategic analysis of the company and the factors that contribute to strengthening sustainable competitive advantages and the ability to absorb external good practices, guidance resulting innovations in
\end{abstract}


process improvements, cost reduction, expansion of business insight and knowledge that strengthen the brainpower for decision-making.

Key words: dynamic capabilities; dynamic markets; organizational learning; competitive advantages; export companies

\section{Introducción}

Frente al dinamismo de los mercados que incrementa la complejidad de las empresas para competir, la perspectiva de las capacidades dinámicas se constituye en un cuerpo robusto de argumentaciones que sostienen que la competitividad depende en mayor medida de la capacidad de las empresas para identificar las transformaciones del entorno y adaptarse a las nuevas condiciones que de este se derivan.

Autores como Teece, Pisano y Shuen (1997) hacen referencia a las capacidades dinámicas como la habilidad de configurar y construir competencias internas y externas direccionadas a los cambios ambientales.

Conforme a la problemática de gestionar los cambios en los mercados, las empresas exportadoras que proyectan un mejor desempeño aprovechan la dotación de capacidades dinámicas para gestionar el conocimiento y a su vez implementar la innovación en los diferentes procesos de creación y mejoras de los productos.

La importancia del tema para las empresas exportadoras se refleja en la necesidad que estas tienen por generar y mantener ventajas competitivas sostenibles en el tiempo. En este sentido, nos planteamos el objetivo de analizar las capacidades dinámicas que han desarrollado varias empresas exportadoras de América Latina, a partir de la revisión de los trabajos de otros autores que han profundizado en la realidad de cada organización. Al hilo del propósito declarado, se aplica un enfoque cualitativo y los métodos teóricos histórico lógico, analítico sintético y el inductivo deductivo.

El aporte de este trabajo está enfocado en proporcionar una mirada sobre las prácticas organizacionales que definen las capacidades dinámicas en las empresas de América Latina, con el fin de que las empresas ecuatorianas las tomen como ejemplo en sus diferentes sectores estratégicos. El conocimiento de estas acciones podría conducir a los empresarios a tomar mejores decisiones respecto a la competencia y habilidad.

La estructura de este trabajo de investigación parte de un marco teórico que destaca la perspectiva de las Capacidades Dinámicas y su relación con la Teoría de Recursos y Capacidades, se refuerza con la recopilación de aspectos conceptuales desde la perspectiva de varios autores. Se contextualizan los beneficios de las capacidades dinámicas en las organizaciones y en las empresas exportadoras. El apartado de resultados comprende el análisis de los principales hallazgos que señalan los trabajos revisados sobre empresas exportadoras latinoamericanas. Finalmente se abordan las conclusiones seguidas de la identificación de futuras líneas de investigación. 


\section{Enfoque de las Capacidades dinámicas}

La obtención de ventajas competitivas ha sido una preocupación constante de las empresas a nivel internacional. Las transformaciones continuas en los mercados han contribuido a incrementar la complejidad por mantenerse en ellos. En este sentido, el dinamismo del entorno marca exigencias que deben ser abordadas con mayor rapidez y flexibilidad por parte de las organizaciones.

Bajo el enfoque de las fuerzas competitivas de Porter (1980), dominante en la década de los ochenta, la explicación de un mejor desempeño de las empresas está relacionado a la existencia de una estructura de mercado caracterizada por cinco fuerzas, las mismas que determinan su atractivo. Así la formulación de las estrategias se orienta por la exploración y selección de las oportunidades o amenazas presentes en el entorno externo para posterior a ello modificar o cambiar los procesos existentes (Donawa y Morales, 2018). Este enfoque ha sido ampliamente aceptado y al mismo tiempo criticado, especialmente por minimizar las potencialidades de las empresas, sus recursos internos y características diferenciadoras.

La Teoría de los Recursos y Capacidades como cuerpo teórico explica que las empresas logran mejores resultados que sus competidores por su dotación de recursos internos identificados como estratégicos, y de capacidades consideradas como habilidades con las que se obtienen nuevos recursos (Grant, 1991). Las capacidades de los directivos pueden desembocar en mejores procesos organizacionales, conocimiento actualizado, y en los colaboradores las capacidades se pueden ver reflejadas en una mejor preparación para el área en que se desempeñan, aspectos que de forma conjunta contribuyen a diseñar y ejecutar nuevas estrategias para alcanzar la eficiencia y efectividad (Then et al., 2014).

El surgimiento de la perspectiva de las Capacidades Dinámicas se encuentra asociado a la Teoría de Recursos y Capacidades, como una extensión a la explicación de la obtención de ventajas competitivas. El término "dinámico" hace referencia a la capacidad de renovar, modificar o cambiar las competencias, las capacidades, los recursos y los modelos de negocio para que se encaminen adecuadamente en los entornos cambiantes (Pavlou y Sawy, 2011).

Las capacidades dinámicas según Teece, Pisano y Shuen (1997) hacen referencia a la habilidad de configurar y construir competencias internas y externas direccionadas a los cambios ambientales. Siguiendo a Teece (2007), las capacidades dinámicas han sido constituidas por las capacidades de seize (posibles inversiones y el evaluar capacidades existentes y emergentes,) sense (identificar oportunidades y monitorear) y reconfiguring (extender, crear y modificar la base de recursos cuando la compañía crece y el mercado cambia).

Entre otras capacidades que se han direccionado como constitutivas de las capacidades dinámicas se encuentra la capacidad de aprendizaje, de coordinación e integración presentes en los estudios de Protogerou, Caloghirou, y Lioukas (2011) y Pavlou y Sawy (2011).

Según Zahra, Sapienza y Davidsson (2006), las capacidades dinámicas están orientadas a cambios estratégicos, a la alineación de las organizaciones con el ambiente y al desempeño de la misma. Cabe resaltar que las capacidades operativas son esenciales para que las capacidades 
dinámicas logren existir, ya que es responsable de la operación del conocimiento (Takahashi, Bulgaco v y Giacomini, 2017).

En base a la literatura revisada se han identificado varias definiciones de las capacidades dinámicas. En efecto, no son pocos los autores que concuerdan con la idea de que el constructo aún atraviesa la etapa de construcción. En la tabla 1 se recogen las perspectivas de varios autores sobre las capacidades dinámicas.

Tabla 1.

Capacidades dinámicas perspectiva de varios autores

Autores Definición de Capacidades dinámicas

Nelson (1991) Rutinas organizativas edificadas sobre una base jerárquica de acuerdo al entorno evolutivo o Schumpeter, hace énfasis a la evolución de las capacidades esenciales.

Collis (1994) Capacidad de desarrollar e innovar con mayor rapidez.

Helfat (1997) Subconjunto de competencias, que permiten a las empresas, crear procesos y productos, además de responder ante las situaciones cambiantes del mercado.

Teece, Pisano \& Habilidad de las empresas, para lograr construir, integrar y sobre todo reconfigurar Shuen (1997) competencias externas como interna, para lograr direccionar la velozmente ante los entornos cambiantes.

Eisenhardt \& Representan una nueva fuente de ventajas competitivas, con la cual las empresas son capaces de Martin (2000) lograr cambios en el entorno.

Zahra \& George Manifiestan que las capacidades están orientadas al cambio lo cual ayuda a que las empresas (2002) logren reemplazar y reconfigurar sus recursos para satisfacer las demandas de los clientes y afrontar las diferentes estrategias de la competencia.

Winter (2003) Capacidades organizativas que se ejercen para crear o modificar (rutinas con un alto nivel) donde las empresas invierten para renovar o modernizar el stock de capacidades.

Vivas (2005) Concluye que las capacidades dinámicas son complejas, los procesos organizacionales con un alto nivel proporcionan condiciones favorables para la modificación y renovación de todos los activos de la organización.

Teece (2007) Capacidades de la empresa (difícil de imitar) que le permiten adaptarse a los diferentes clientes cambiantes además de aprovechar las diferentes oportunidades tecnológicas; incluye también la capacidad de configurar el contexto que se desenvuelven (desarrollo de nuevos procesos, productos y modelo de negocios factibles.

Kim, Shin, Kyu, Capacidades de la empresa que posee para cambiar es decir adaptarse, mejorar o reconfigurar \& Geun(2011) los procesos que posee en relación a la competencia, por medio de reducción de costos y el aprendizaje estructural.

Garzón (2015)

Es la potencialidad que una empresa posee para crear nuevos saberes organizacionales (recreación, renovación, incremento, mejora, protección y reconstrucción de sus corecompetences) cuyo fin es el de responder favorablemente a los diferentes cambios en el mercado y la tecnología.

Fuente: Elaboración del autor

De acuerdo al criterio de los autores señalados, las capacidades dinámicas involucran aquellos cambios estratégicos reactivos o proactivos (habilidades y competencias) que facilitan y 
direccionan los diferentes procesos a una innovación, modificación, creación o reconfiguración de los diversos recursos y capacidades. En este sentido, las capacidades dinámicas que desarrollan las organizaciones posibilitan realizar ajustes ante el dinamismo que presenta el entorno.

\section{Capacidades dinámicas en las organizaciones.}

La globalización ha generado grandes cambios en los mercados, la tecnología empleada, la transmisión de la información, la forma de comunicación entre los participantes (competidores, proveedores, compradores) es solo una muestra de los aspectos que hacen más complejo el escenario de la competencia para las empresas. La incertidumbre se suma al conjunto de elementos que deben considerar los directivos para la toma de decisiones.

De acuerdo a Arteaga, Intriago y Mendoza (2016), en la actualidad, las empresas con relativo éxito en los mercados se destacan por desarrollar ventajas competitivas sostenibles enfocadas en gestionar las capacidades en términos de conocimiento, información y procesos. Así, un ejemplo de ello se identifica en las capacidades esenciales para obtener, interpretar información y procesarla con el fin de desarrollar nuevos productos, además de evaluar sus límites técnicos como: viabilidad económica y posibilidad de fabricación (Fayos, Calderón y Almanzar, 2017). Al hilo de los expuesto, la capacidad de absorción del conocimiento, el aprendizaje y la innovación conducen a un aumento en el valor de las organizaciones que les permite mejorar su posicionamiento (Mendoza, 2018).

En este sentido, las capacidades dinámicas desarrolladas a nivel organizacional, como competencias gerenciales (Valencia, 2015) se visualizan como un aprendizaje continuo articulado a la identificación de cambios y la necesidad de adecuaciones de la estrategia empresarial (Jurksiene y Pundziene, 2016). De acuerdo a Wohlgemuth y Wenzel (2016), el aprendizaje colectivo es el que representa mayor relevancia para el desarrollo de las capacidades dinámicas.

Las empresas exportadoras, independientemente de su origen o tamaño, se enfrentan en mercados altamente competitivos donde son visibles los cambios desarrollados para competir. De allí que la utilidad de las capacidades dinámicas, de acuerdo con el criterio de Acosta et al. (2019), se reflejan como la capacidad de utilizar el aprendizaje como un flujo para actualizar las habilidades y competencias internas en una reconstrucción continua a lo largo de la vida empresarial. Por consiguiente, en las empresas exportadoras, las capacidades permiten abordar nuevos mercados, y reducir la probabilidad de efectos negativos de las amenazas a nivel empresarial e incrementar las posibilidades de aprender y adoptar las enseñanzas de los nuevos mercados (Krzakiewicz y Cyfert, 2014).

Cabe destacar que una vertiente del análisis se ha enfocado en discutir que la relevancia de las capacidades dinámicas para las organizaciones se encuentra asociada al tipo de ambiente en que éstas se desenvuelven, pudiendo ser altamente dinámico o moderado (Teece, Pisano y Shuen, 1997; Eisenhardt y Martin, 2000; Peteraf y Stefano, 2013). 
En los mercados moderadamente dinámicos, los cambios suceden con cierta frecuencia, son más o menos lineales y previsibles. Las capacidades dinámicas en estos mercados dependen mayormente del conocimiento existente y de aquellas rutinas que den paso al aprovechamiento de las oportunidades (Eisenhardt y Martin, 2000). Los mercados altamente dinámicos relacionados con el comercio internacional se caracterizan, por ejemplo, por cambios en regulaciones sanitarias, normativas de acceso, aspectos que las empresas exportadoras con capacidad de adaptación pueden gestionar con respuestas innovadoras, como las alianzas estratégicas (Miranda, 2015) y mejorar su conocimiento del entorno (Zapata y Mirabal, 2018).

\section{Metodología}

En función del objetivo planteado que consiste en analizar y caracterizar las capacidades dinámicas de empresas latinoamericanas, se aplican métodos teóricos para describir las prácticas organizacionales que reflejan dichas capacidades. El presente trabajo se desarrolla bajo un enfoque cualitativo. En este sentido, la investigación es de carácter descriptivo, partiendo de fuentes secundarias, seleccionamos una muestra de 9 trabajos de investigación referidos a las manifestaciones de las capacidades dinámicas en empresas de varios sectores de Latinoamérica.

Los métodos teóricos empleados corresponden al método histórico lógico, método analítico-sintético y el inductivo deductivo. Consideramos que estos métodos son acordes al propósito de realizar un análisis de la evolución de las prácticas organizacionales en contextos latinoamericanos, que finalmente se manifiestan en capacidades dinámicas.

Los estudios de Lane y Lubat-Kin (1998) refieren a las capacidades dinámicas como "la habilidad de la empresa para asimilar y aplicar el conocimiento proveniente de otra empresa". Estas capacidades se engloban a través de 4 coordenadas, siendo estas: la estructura organizativa, la similitud entre empresas, la lógica dominante y el conocimiento base. De igual forma, Zou et al. (2018) realizaron un estudio en el que se determinó a la edad de la empresa y el tamaño, como puntos clave para alcanzar el éxito en las capacidades dinámicas, en donde las pequeñas empresas alcanzan logros positivos en comparación de las grandes empresas y empresas maduras. De este estudio, se relaciona a los trabajos objeto de estudio.

\section{Resultados}

\section{Las capacidades dinámicas en las empresas exportadoras latinoamericanas}

Las empresas exportadoras se encuentran inmersas en un continuo proceso de aprendizaje al tener que identificar los requisitos de la demanda extranjera y desarrollar aspectos visibles de valor agregado para la satisfacción de su mercado, lo que exige a su vez mejorar la calidad (Gerschewsky, 2011; Atkin, Khandelwal y Osman, 2014), reevaluar las estrategias en el ámbito operativo y administrativo (Beleska-Spasova, 2014).

Con el análisis de los estudios previos sobre empresas latinoamericanas de características heterogéneas, pretendemos identificar las capacidades dinámicas que desarrollan para fortalecer sus estrategias competitivas, en ambientes de continuo cambio. Bajo este preámbulo, en la Tabla 
2 se recoge una muestra de nueve estudios relacionados con las capacidades dinámicas de empresas que actúan en el campo de las exportaciones, destacando los principales hallazgos.

Tabla 2.

Las capacidades dinámicas en empresas de América Latina

Autor/año $\quad$ Muestra/País $\quad$ Principales resultados

\begin{tabular}{ll}
\hline Matos, & 498 empresas \\
Fernández, & $\begin{array}{l}\text { manufactureras, } \\
\text { exportadoras de Brasil } \\
\text { Oliveira (2015) }\end{array}$
\end{tabular}

Las exportadoras brasileñas del sector optan por innovaciones con mayor frecuencia centradas en los procesos, mas no en las mejoras del producto. La capacidad dinámica del sector exportador brasileño se refleja en la adaptación de procesos y la reducción de costos, tomando en consideración la innovación en el sector que conduce a la reducción de costos en procesos para ingresar a mercados internacionales con precios promedios más bajos.

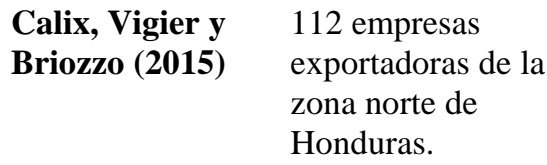

Calderón y 7 empresas

Kolbe (2016) exportadoras agroalimentarias, Venezuela .

Camargo, Díaz, 249 Pymes Velandìa y Evaristo (2017) exportadoras del sector industrial, Colombia.

$\begin{array}{ll}\text { Rodríguez, } & 119 \text { empresas } \\ \text { Wise y } & \text { exportadoras, } \\ \text { Martínez (2013) } & \text { México. }\end{array}$

$\begin{array}{ll}\text { Escandón, } & 297 \text { empresas } \\ \text { Rodríguez y } & \text { manufactureras, } \\ \text { Hernández } & \text { Colombia. }\end{array}$
(2013)
Las capacidades dinámicas de las empresas en estudio se articulan al capital intelectual, la gestión del conocimiento, la experiencia y el recorrido internacional. Las exigencias de los mercados, por el aspecto normativo como por el lado de los consumidores, aceleran el crecimiento de la competitividad. Se destaca una relación positiva entre la capacidad de las empresas para adoptar estrategias complementarias, flexibilidad organizacional y su habilidad para exportar.

Los autores del estudio distinguen en el sector las capacidades dinámicas de tipo general y específico. Entre las capacidades generales se identifica el conjunto de habilidades para la adaptación a los cambios del mercado, la proyección de la empresa en el futuro, la acumulación y asimilación de conocimiento y aprendizaje.

Las capacidades específicas se reflejan en la forma de establecer relaciones comerciales, el cuidado y potenciación de la marca, además de la gestión de las comunicaciones.

Las Pymes exportadoras de la industria metalmecánica orientan su capacidad dinámica al desarrollo de estrategias externas de búsqueda y cooperación con los stakeholders o partes interesadas. Por otro lado, las estrategias internas de las pymes se basan en crear ambientes y proveer recursos propicios al capital humano para el desarrollo del proceso innovador.

Las empresas exportadoras mexicanas reflejan sus capacidades dinámicas en la innovación y la orientación empresarial. Se destaca la capacidad de absorción desarrollada de la mano del conocimiento del mercado y de los clientes, equivalente a la capacidad de exploración para capturar el conocimiento externo útil. Como complemento, la capacidad de explotación se identifica con la adaptación a las nuevas condiciones mediante la flexibilidad de procesos y la capacidad de innovación para responder de forma satisfactoria a los cambios detectados en la demanda.

Las empresas de este estudio se distinguen por su acelerado proceso de crecimiento apoyado en la creación de alianzas, de cooperación y aprendizaje organizacional. Impulsando capacidades como el desarrollo de relaciones con clientes, desarrollan ventajas competitivas a través del compartimiento de la información y mitigación de riesgos; se caracterizan por el apoyo mutuo entre las empresas fomentando una buena relación transversal con el sector externo. Como mecanismo interno buscan siempre la creación del conocimiento propio a través de los mecanismos de aprendizaje. 


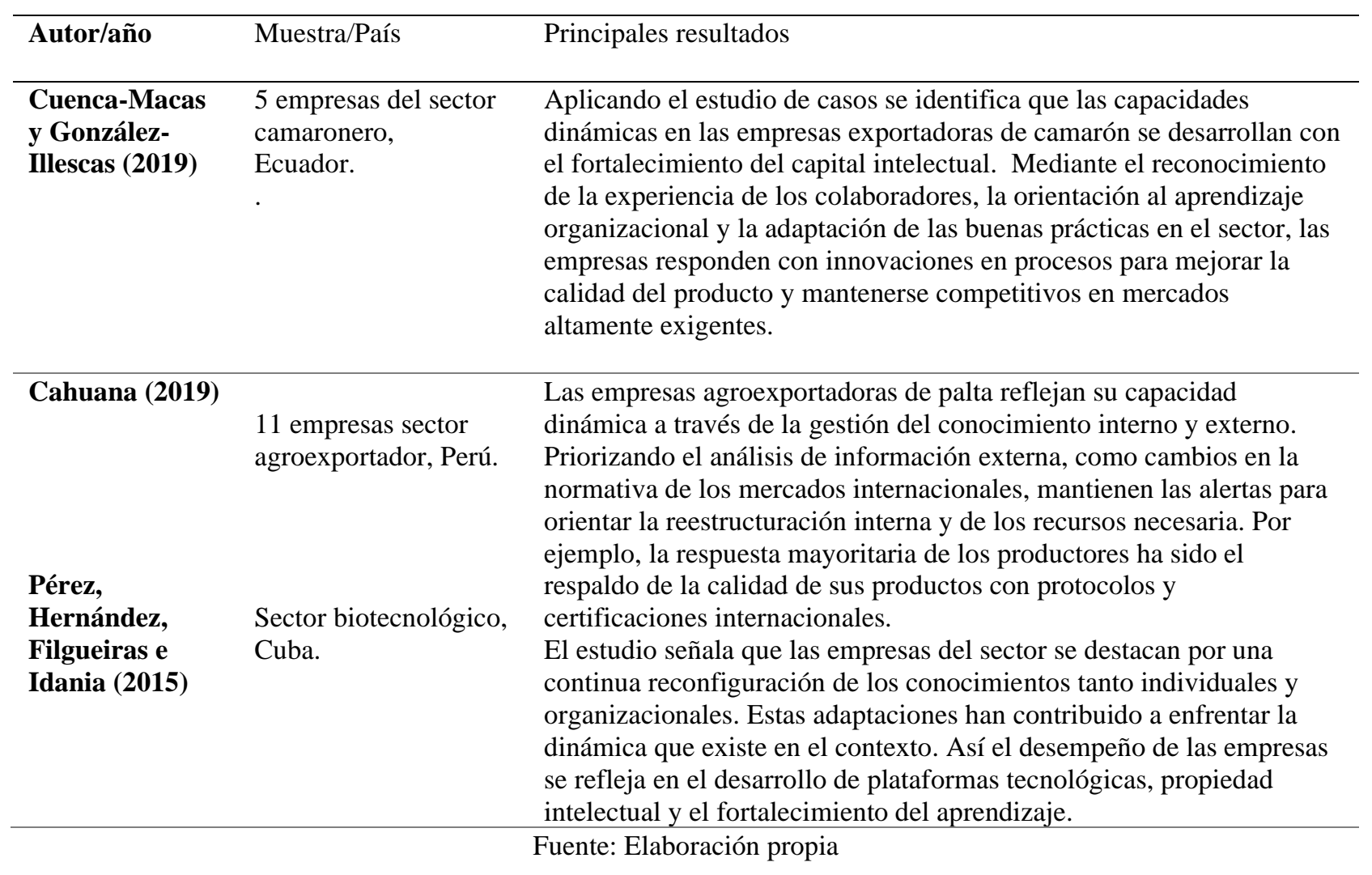

Entre las revisiones realizadas se evidencia que las capacidades dinámicas representan un aprendizaje organizacional que incorpora los cambios del entorno, siendo útil para esbozar estrategias (Alinaghian, 2012). Así, siguiendo el criterio de Fayos, Calderón y Almanzar (2017), la información oportuna de los cambios que podrían afectar a la empresa permitiría reducir pérdidas, optimizar operaciones, procesos internos, como aprovechar oportunidades derivadas de la nueva situación del mercado. En este sentido, las capacidades dinámicas permiten a las empresas identificar y dimensionar las nuevas oportunidades, así como renovar la base de activos existentes.

Las capacidades dinámicas del sector exportador de los diferentes países, les ha permitido gestionar el conocimiento e implementarlo en temas de innovación tanto en procesos sistemáticos como en procesos de creación y mejoras en los productos. La visión empresarial, les ha permitido crecer competitivamente consolidándose en capacidades de networking y adaptación continua ante los mercados cambiantes.

En Ecuador, de acuerdo con Cuenca-Macas y González-Illescas (2019), el sector camaronero representa un interesante caso de estudio, destacando su época de crisis y auge. Desde la perspectiva de las prácticas organizacionales, las capacidades dinámicas se encuentran asociadas a la necesidad de responder de forma coherente y con rapidez frente a los cambios de las normativas sanitarias y de calidad que determinan los mercados receptores. Del mismo estudio se desprende que las capacidades dinámicas se desarrollan en el marco de la dirección del conocimiento interno y externo, cuyo papel se convierte en estratégico para conducir a la empresa a generar ventajas competitivas sostenibles en los mercados internacionales. 


\section{Conclusiones}

El enfoque de las capacidades dinámicas refleja la evolución del análisis estratégico de la empresa y de los factores que contribuyen a fortalecer las ventajas competitivas sostenibles.

Los cambios constantes en el entorno marcan grandes desafíos a las empresas. La velocidad y coherencia de respuestas definirán en parte el éxito frente a las exigencias de los consumidores y las fuerzas del mercado. En este sentido, las capacidades dinámicas se visualizan como la capacidad de adaptación a los entornos altamente cambiantes lo que engloba flexibilidad, cambios estratégicos, modificación, creación, reconfiguración de los diversos recursos, procesos y habilidades colectivas que las organizaciones ponen en práctica para alcanzar sus objetivos.

Por otro lado, si bien el dinamismo del entorno influye en el ritmo de desarrollo de las capacidades dinámicas de las empresas que priorizan la información del ambiente externo, la existencia de entornos con menor turbulencia, como el caso de mercados estables, no es sinónimo de empresas con un menor desarrollo de las capacidades, pues no se constituye en condición para su desarrollo. El análisis del entorno externo implica la adquisición de nuevos conocimientos, junto al aprovechamiento de las condiciones del ambiente interno y las adaptaciones necesarias, reflejan un aprendizaje continuo.

En lo que respecta a las empresas exportadoras latinoamericanas las capacidades dinámicas se manifiestan en la capacidad de absorber las buenas prácticas externas, la orientación a innovaciones que se derivan en mejoras de procesos, reducción de costos, ampliación de una visión empresarial y de conocimientos que fortalecen el capital intelectual para la toma de decisiones. En este contexto cabe destacar la creación de ambientes de colaboración a través de la gestión de alianzas estratégicas que han conducido a las empresas hacia una mejora en el aprendizaje organizacional que se ha derivado en el fortalecimiento de capacidades para enfrentar la competencia en los mercados dinámicos.

Finalmente, con relación a futuras líneas de investigación, reconociendo las limitaciones de la presente al tener un enfoque cualitativo a partir de información secundaria, consideramos oportuno pasar a una siguiente fase, bajo un enfoque cuantitativo, sustentado

en las percepciones y experiencia de los empresarios ecuatorianos, mediante entrevistas y cuestionarios que permitan recoger las variables que caracterizan las prácticas organizacionales de las empresas exportadoras comprendidas en la creación y desarrollo de capacidades dinámicas.

Sin embargo las empresas ecuatorianas a pesar que aún se encuentran en grandes competencias con países desarrollados, han apostado por la innovación y la visión empresarial para resaltar sus capacidades dinámicas, no obstante, este estudio permitirá que los empresarios de las empresas más competitivas del país comiencen a fortalecer cada uno de sus sistemas productivos hasta lograr las practicas organizaciones de calidad. 


\section{Bibliografía}

Acosta , J., Plata, K., Puentes, D., \& Torres, M. (2019). Influencia de los recursos y capacidades en los resultados financieros y en la competitividad empresarial: una revisión de la literatura. I+D. Revista de Investigaciones, 13(1), 125-134.

Arteaga, H., Intriago, D., \& Mendoza, K. (2016). La ciencia de la administración de empresas. Revista Científica Dominio de las Ciencias, 2(4), 421-431.

Atkin, D., Khandelwal, A., \& Osman, A. (2014). Exporting and Firm Performance: Evidence from a Randomized Trial. EconPapers(10276), 20-30.

Calderón, H., \& Kolbe, D. (2016). Capacidades dinámicas en el proceso de internacionalización de las empresas de distribución de productos agroalimentarios. Economía Agraria y Recursos Naturales, 16(2), 5-32.

Calix, C., Vigier, H., \& Briozzo, A. (2015). Capital intelectual y otros determinantes de la ventaja competitiva en empresas exportadoras de la zona norte de Honduras. Suma de negocios, 6(14), 130-137.

Camargo, G., Díaz, R., Velandìa, G., \& Evaristo, N. (2017). Capacidad dinámica de innovación en las PyME exportadoras metalmecánicas en Colombia. Espacios, 38(58), 16.

Collis, D. (1994). Research note: How valuable are organizational capabilities? Strategic Management Journal, 15, 143-152.

Cahuana, M. (2019). Exportaciones de palta Hass y desarrollo del agro no tradicional, Región La Libertad (2010 - 2016). Neumann Business Review, 5(1), 62 - 77.

Cuenca, J., \& González, M. (2019). Gestión del capital intelectual e innovación. Percepción de los exportadores de camarón. Revista Ciencia UNEMI, 12(3O), 160 - 172.

Dávila, J. (2013). Capacidades organizacionales: dinámicas por naturaleza. Cuadernos de Administración, 26(47), 11-33.

Donawa, Z., \& Morales, E. (2018). Fuerzas competitivas que moldean la estrategia en la gerencia del sector Mi PyME del distrito de Santa Marta -Magdalena, Colombia. Revista EAN(38), 97-108.

Eisenhardt, K., \& Martin, J. (2000). Dynamic capabilities: what are they? Strategic Management Journal, 21.

Escandón, D. M., Rodríguez, A., \& Hernández, M. (2013). La importancia de las capacidades dinámicas en las empresas born global colombianas. Cuadernos de Administración, 26(47), 141-164.

Fayos, T., Calderón, H., \& Almanzar, M. (Abril de 2017). Las capacidades dinámicas en la internacionalización de las empresas y cooperativas agroalimentarias integradas en clusters. CIRIEC-España, Revista de Economía Pública, Social y Cooperativa(89), 4-31.

Garzón, M. (2015). Modelo de Capacidades Dinámicas. Revista Dimensión Empresarial, 13(1), 111-131.

Grant, R. (1991). The Resource-Based Theory of Competitive Advantage: Implications for Strategy Formulation. California Management Review, 33,(3), 114-135.

Helfat, C. (1997). Know-how and asset complementary and dynamic capability accumulations: The casa of R5D. Strategic Management Journal, 18(5), 393-360.

Helfat, C., \& Peteraf, M. (2015). Managerial Cognitive Capabilities and the Microfoundations of Dynamic. Strategic Management Journal, 36(6), 831-850.

Henderson, R., \& Cockburn, I. (1994). Measuring. Strategic Management Journal, 42(1), 63-84.

Esta obra se comparte bajo la licencia Creative Common Atribución-No Comercial 4.0 International (CC BY-NC 4.0) 
Jurksiene, L., \& Pundziene, A. (2016). The Relationship Between Dynamic Capabilities and Firm Competitive Advantage: The Mediating Role of Organizational Ambidexterity. European Business Review, 28(4), 431-448.

Krzakiewicz, K., \& Cyfert, S. (2014). Thee Strategic Dimension of the Dynamic Capabilities of Enterprises. Management, 18(2), 4-10.

Kim, G., Shin, B., Kyu, K., \& Geun, H. (2011). IT Capabilities, Process-Oriented Dynamic Capabilities, and Firm Financial Performance. Journal of the Association for Information Systems, 12(7), 487-517.

Kogut, B., \& Zander, U. (1992). Knowledge of the firm, combinative capabilities, and the replication of technology. Organizational Science, 3(3), 383-397.

Matos, A., Fernández, F., Braga, M., \& Oliveira, J. (2015). Capacidades, inovação e desempenho geral das empresas brasileiras exportadoras. Adm. Mackenzie, 16(3), 76-108.

Mendoza, J. (2018). Capacidades dinámicas: Un análisis empírico de su naturaleza. $M L S$ Educational Research, 2(2), 193-210.

Miranda, J. (2015). El modelo de las capacidades dinámicas en las organizaciones. Investigación Administrativa, 44(116), 82-83.

Pavlou, P., \& Sawy, O. (2011). Understanding the elusive black box of dynamic capabilities. Decision Sciences Journal, 42(1), 239-273.

Peteraf, M., Stefano, G., \& Verona, G. (2013). The elephant in the room of dynamic capabilities: Bringing two diverging conversations together. Strategic Management Journal, 34(12).

Pérez, M., Hernández, G., Filgueiras, M., \& Idania, C. (2015). Capacidad dinámica de aprendizaje organizacional en empresas biotecnológicas cubanas de alta tecnología. Ingeniería Industrial, 36(2), 212-223.

Porter, M. (1980): Estrategia competitiva. Técnica para el análisis de los sectores industriales y de la competencia, Compañía Editorial Continental, México.

Protogerou, A., Caloghirou, Y., \& Lioukas, S. (2011). Dynamic capabilities and their indirect impact on firm performance. Industrial Corporate Change, 21(3), 615-647.

Rodriguez CM, Wise JA \& Martinez CR (2013) Strategic capabilities in exporting: an examination of the performance of Mexican firms. Management Decision 51(8): 16431663.

Schumpeter, J. 1. (2012). The theory of economic development: An Inquiry into Profits, Capital, Credit, Interest and the Business Cycle, Publishers. Journal of Comparative Research in Anthropology and Sociology, 3(2), 2068 - 0317.

Takahashi, A., Bulgacov, S., \& Giacomini, M. (2017). Capacidades dinâmicas, capacidades operacionais (educacional-marketing) e desempenho. Revista Brasileira de Gestão de Negócios, 19(65).

Teece, D. (2007). Explicating dynamic capabilities: The nature and microfoundations of enterprise performance. Strategic Management Journal, 28(13), 40-60.

Teece, D. J., Pisano, G., \& Shuen, A. (Aug de 1997). Dynamic Capabilities and Strategic Management. Strategic Management Journal, 18(7),20-35.

Then, L., Pimentel, S., Olivero, P., Soto, A., Luna, A., Cruz, G., y otros. (2014). Análisis de las fuerzas competitivas de Porter en el sector de promoción inmobiliaria del gran Santo Domingo. Ciencia y Sociedad, 39(3), 441-476.

Valencia, M. (2015). Capacidades dinámicas, innovación de producto e aprendizaje organizacional en pymes del sector cárnico. Revista Ingeniería Industrial, XXXVI(3), 297-305. 
Vivas, S. (2005). Competitive advantage and strategy formulation the key role of dynamic capabilities. Management Decisión, 43(5-6).

Winter, S. (2003). Understanding Dynamic Capabilities. Strategic Management Journal, 24(10), 991-995.

Wohlgemuth, V., \& Wenzel, M. (2016). Dynamic capabilities and routinization. Journal of Business Research, 69(5), 1944-1948.

Zahra, S., \& George, G. (2002). Absorptive Capacity: A Review, Reconceptualization and Extension. Academy of Management Review, 27(2), 213-240.

Zahra, S., Sapienza, H., \& Davidsson, P. (2006). Entrepreneurship and dynamic capabilities: A review, model and research agenda. Journal of Management Studies, 43(4), 50-70.

Zapata, G., \& Hernández, A. (2014). Origen de los Recursos y Ventajas Competitivas de las Organizaciones:Reflexiones Teóricas. Revista Venezolana de Gerencia (RVG), 19(68), 735-759.

Zapata, G., \& Mirabal, A. (2018). Capacidades Dinámicas de la Organización: Revisión de la Literatura y un Modelo Propuesto. Investigación administrativa, 47(121), 7-14. 